\title{
Geometrically Accurate Topology-Correction of Cortical Surfaces Using Nonseparating Loops
}

\author{
Florent Ségonne*, Jenni Pacheco, and Bruce Fischl
}

\begin{abstract}
In this paper, we focus on the retrospective topology correction of surfaces. We propose a technique to accurately correct the spherical topology of cortical surfaces. Specifically, we construct a mapping from the original surface onto the sphere to detect topological defects as minimal nonhomeomorphic regions. The topology of each defect is then corrected by opening and sealing the surface along a set of nonseparating loops that are selected in a Bayesian framework. The proposed method is a wholly self-contained topology correction algorithm, which determines geometrically accurate, topologically correct solutions based on the magnetic resonance imaging (MRI) intensity profile and the expected local curvature. Applied to real data, our method provides topological corrections similar to those made by a trained operator.
\end{abstract}

Index Terms-Homotopy, human cerebral cortex, nonseparating loop, Reeb graph, segmentation, topology.

\section{The CorticAl Reconstruction Problem}

$\mathbf{T}$ HE human cerebral cortex is a highly folded ribbon of gray matter (GM) that lies inside the cerebrospinal fluid (CSF) and outside the white matter (WM) of the brain. Locally, its intrinsic "unfolded" structure constitutes a 2-D sheet, which is several millimeters thick. In the absence of pathology and assuming that the midline hemispheric connections are artificially closed, each cortical hemisphere can be viewed as a simply connected 2-D sheet of neurons that carries the simple topology ${ }^{1}$ of a sphere ${ }^{2}$ [Fig. 1(a)].

Manuscript received September 6, 2006; revised October 11, 2006. This work was supported in part by the National Center for Research Resources under Grant P41-RR14075, Grant R01 RR16594-01A1, and the NCRR BIRN Morphometric Project BIRN002, U24 RR021382, in part by the National Institute for Biomedical Imaging and Bioengineering under Grant R01 EB001550, in part by the National Institute for Neurological Disorders and Stroke under Grant R01 NS052585-01, and in part by the Mental Illness and Neuroscience Discovery (MIND) Institute. Asterisk indicates corresponding author.

*F. Ségonne is with the CERTIS Laboratory, ENPC, 19 rue NobelCité Descartes, Champs-sur-Marne 77455, France (e-mail: florent. segonne_ieeetmi@m4x.org).

J. Pacheco is with the Computational Core at the Athinoula A. Martinos Center for Biomedical Imaging, Massachusetts General Hospital, Harvard Medical School, Charlestown, MA 02129 USA.

B. Fischl is with the Computational Core at the Athinoula A. Martinos Center for Biomedical Imaging, Massachusetts General Hospital, Harvard Medical School, Charlestown, MA 02129 USA and also with the CSAIL, Massachusetts Institute of Technology, MA 01239 USA.

Color versions of one or more of the figures in this paper are available online at http://ieeexplore.iee.org.

Digital Object Identifier 10.1109/TMI.2006.887364

\footnotetext{
${ }^{1}$ In mathematical terms, two surfaces have the same topology if they are homeomorphic.

${ }^{2}$ The true topology of the gray/white surface is not one of a sphere, as a result of the midline connections between the left and right hemisphere, such as the anterior and the posterior commisures.
}
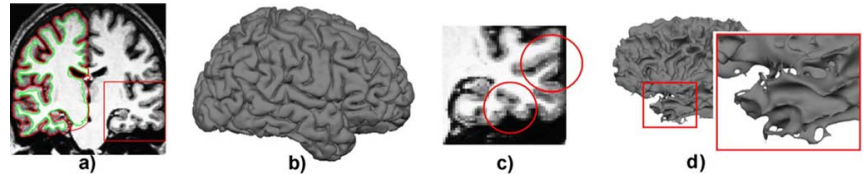

Fig. 1. (a) The human cerebral cortex is a highly folded ribbon of GM that lies inside the cerebrospinal build and outside the WM of the brain. The green surface represents the interface between WM and GM, and the red surface (i.e., the pial surface) models the interface between GM and CSF. When the midline connections between the left and right hemisphere are artificially closed, these two surfaces have the topology of a sphere. (b) Three-dimensional rendering of the highly folded pial surface. Opposite regions of a sulcus are often contiguous. (c) As a result of the partial volume effect, it is difficult to distinguish opposite banks of the GM from one another. (d) Segmentation algorithms that do not constrain the topology often create cortical segmentations with certain topological defects (i.e., handles). Close-up of a topologically incorrect gray/white surface representation.

Recently, there has been a research focus on the extraction of accurate and topologically correct models of the brain surface. The development of these algorithms greatly facilitates the analysis of cortical data [1], [2], and alleviates many problems of the 3-D embedding space (such as the underestimation of true cortical distances or the overestimation of cortical thicknesses). Certain clinical and research applications depend crucially on the accuracy and correctness of the representations: visualization [1]-[3], spherical coordinate system and surface-based atlases [1], [2], [4]-[8], shape analysis [8]-[13], surface-based processing of functional data [1], and intersubject registration [5], [14], [15], among others.

Many recent segmentation algorithms for neuroimaging data are able to identify and precisely locate diverse brain structures, although typically without ensuring the validity of the final topology (i.e., that of a sphere). Magnetic resonance images (MRIs) often contain various artifacts (e.g., image noise, image intensity inhomogeneity or nonuniformity, caused by RF inhomogeneities, partial volume averaging effects, and subject motion) that are difficult to predict and model. In the case of cortical segmentations, partial volume effects makes the accurate location of the surface of the cortex particularly difficult (Fig. 1). Because of its highly folded nature, opposite banks of a sulcus often appear connected, and small gaps between adjacent folds of the neocortical GM become invisible in the finite resolution MRIs [Fig. 1(b) and (c)]. Conversely, thin strips of WM often appear darker due to neighboring $\mathrm{GM},{ }^{3}$ and the interface between GM and WM is often pierced by incorrectly identified GM in many segmentations [Fig. 1(d)]. These topological defects in the segmentation are hard to detect and correct

${ }^{3}$ Particularly in the case in the parahippocampal gyrus, where the WM is only a voxel or two thick. 


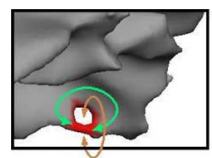

a)

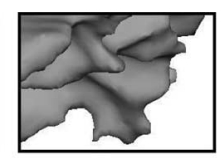

b)

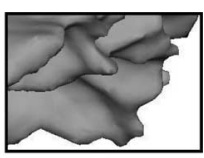

c)
Fig. 2. Methods that aim to correct the topology of a segmentation by minimizing the number of modifications in the segmentation might not produce valid corrections. (a) A topological defect is identified in red on the original topologically incorrect cortical surface. A topological defect can be interpreted as a handle (in green) or, equivalently, as a hole (in brown). (b) Cutting the handle: an incorrect topological correction based only on the size of the defect. In this case, cutting the handle corresponds to a "smaller" modification of the surface than filling the corresponding hole. However, this topological correction is not the right one and brings about an inaccurate cortical representation. (c) Filling the hole: correct topological correction realized by a trained operator. Note that the correction filling a hole is equivalent to cutting a "background" handle. Another point to note is that the correct modification cannot be determined solely from the surface- - the intensity volume is required in order to assess the correct location of the true surface.

retrospectively, with the automatic extraction of accurate and topologically correct cortical surfaces remaining a challenge.

\section{State of the ART In Segmentation Under Topological CONSTRAINTS}

Segmenting under topological constraints is difficult. Segmentation algorithms (see [16]-[18] for some good reviews), which operate on the intensity or variations of the texture of the image, are sensitive to the artifacts produced by the image acquisition process. Most often, segmentation techniques that do not integrate any topological constraints generate segmentations that contain small deviations from the true anatomy of the structures of interest. In the case of cortical segmentations, these deviations can form handles [or holes, which are topologically equivalent-Fig. 2(a)] that erroneously connect parts of the volume.

Integrating topological constraints into the segmentation process significantly complicates the task. Topology is both a global and a local property; small and local modifications of a geometric shape can change its global connectivity. At the same time, topology is intrinsically a continuous concept, and topological notions are difficult to adapt into a discrete framework. For these reasons, the number of techniques available and applicable to the segmentation of images is quite limited.

Methods creating topologically correct cortical models can be divided into two categories: topologically constrained segmentation methods that directly incorporate topological constraints into the segmentation process, and retrospective topology correction techniques that aim to correct the topology of alreadysegmented images. For a complete review, refer to [19].

\section{A. Topologically Constrained Segmentation}

Approaches that integrate the topological constraint directly into the segmentation process have the advantage of allowing the user to explicitly specify the topology of the final segmentation. A model, carrying the desired topology, is iteratively deformed onto the cortical surface while preserving its topology. To this end, active contours [20] (explicit representations [3], [21]-[26] and implicit representations [27]-[29]), digital models [30]-[33], and registration methods [34]-[36] have proven to be extremely useful. Unfortunately, the energy functionals driving the deformation are highly nonconvex, and attaining the desired final surface most often requires an initialization of the model that is close to its final configuration. In addition, local topological constraints can easily cause large geometrical inaccuracies that are difficult to identify and correct.

\section{B. Retrospective Topology Correction}

Recently, new approaches have been proposed to retrospectively correct the topology of an already segmented image. These techniques, which do not enforce any topological constraints into the segmentation process, focus on more accurate models. Many segmentation techniques, using local intensity, prior probabilities, and/or geometric information, while giving no consideration to topology, generate accurate cortical surfaces with few topological inconsistencies. Retrospective techniques aim to identify and correct these defects in order to produce geometrically accurate topologically correct surfaces.

Most retrospective methods do not make full use of all available information. They assume that the topological defects in the segmentation are located at the thinnest parts of the volume and aim to correct the topology by minimizing the number of modifications in the original segmentation [37]-[40]. These methods, which rely on the accuracy of the initial segmentations, often produce accurate topological corrections. Yet, the resulting solutions are rarely geometrically accurate as they do not correspond to the ones that a trained operator would make. Additional information, such as the expected local curvature or the local intensity distribution, may lead to different corrections, i.e., possibly comparable to the ones a trained operator would make. This is illustrated by Fig. 2.

Only a handful of techniques have been proposed to integrate additional information into the topological correction process [19], [41]-[43]. Yet, for a given topological defect, these methods fail to generate multiple potential solutions that are requisite for selecting the expected correction. In fact, they produce only two candidate solutions. Two potential solutions that correct a handle are cutting the handle or filling the corresponding hole (i.e., cutting the "background" handle). However, the exact location of these potential corrections (i.e., the resulting shape of the potential corrections) is often determined by criteria that ignore the underlying MRI intensity profile and/or the local curvature, which means that the resulting corrections are never optimized relative to these parameters. Other solutions, such as the ones a trained operator makes, do not arise. This problem arises when the proposed framework does not examine multiple candidate solutions. Fig. 3 illustrates the difficulty of finding the correct solution when the topological defect is complex.

To our knowledge, only one approach [44] has been proposed to achieve optimal topological corrections integrating a wide range of information available in the image. Similar to the approach developed by Fischl et al. [42], we construct a mapping from the initial triangulation onto the sphere and locate topological defects as locally noninvertible regions. We introduce a genetic algorithm to explore the space of possible surface retessellations and to select an optimal configuration. However, this approach suffers from several drawbacks (described in 


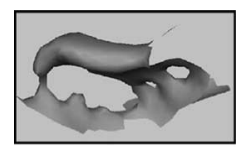

a)

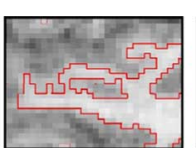

b)

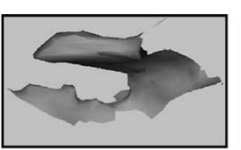

c)

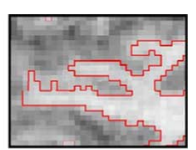

d)
Fig. 3. Some topological defects are complex and extremely hard to correct. Existing methods (with the exception of [44]) only produce a few potential topological solutions, with the chosen solution rarely the expected one. (a) A complex topological defect comprised of three handles. (b) One sagittal MRI slice of the topological defect, illustrating the complexity of the underlying MRI intensity profile. (c) Proper solution realized by a trained operator. (d) sagittal cut of the MRI volume showing the location of the surface of the corrected defect.

Section II-C), and topological corrections are not always the desired ones. We then propose several extensions that we believe resolve these issues, yielding an accurate and automated method for generating topologically correct models of the human cerebral cortex.

\section{Approach}

For a given topological defect, the MRI intensity profile contains important information regarding the location and position of the potential topological correction. The corrected surface should be located at the border of the WM and GM, with WM inside and GM outside the surface. In addition, the smoothness of the corrected defect should be comparable to the smoothness of the remainder of the cortical surface. Incorporating this information into the topology correction procedure can exclude classes of inaccurate solutions, leading to a significantly more robust and accurate technique.

Our method is based on the notion that if a surface has spherical topology it cannot contain any noncontractible curves (i.e., handles). More technically, this can be restated as "every closed 2-manifold that is homeomorphic to a sphere is simply connected" [45]-[47]. This of course is the 2-D analog of the well-known Poincare conjecture (one of the seven Millennium Prize Problems selected by the Clay Mathematics Institute [48]), which appears to have been recently proven by G. Perelman [49]-[51].

The approach follows from our previous work [44]. Although the previous method often yields valid solutions, it has some limitations.

- Spherical mapping: the space of potential retessellations depends on the initial mapping. In [19], we address this problem by generating an array of distinct mappings. However, the resulting procedure is time-consuming and not well-adapted to large topological defects, such as the ones that often arise in the medial temporal lobe and temporal poles.

- Self-intersections: the retessellation procedure, which does not directly guarantee that the final surface does not intersect itself, creates an excessive number of self-intersecting candidate solutions that must be discarded.

- Numerical stability: the method relies on floating-point arithmetic to constrain the topology of the final surface, a formulation in which rounding errors can be problematic. More specifically, the topology is constrained onto the sphere by detecting edge-edge intersections during the retessellation process. By the inherent nature of the spherical mapping, which aims to minimize regions with negative areas (i.e., negative Jacobian), topological defects correspond to extremely dense regions, with vertices potentially being as close as $10^{-5} \mathrm{~mm}$. At this scale, floatingpoint rounding errors can occur and bring about "catastrophic" results: a nondetected intersection engendering a topologically inconsistent retessellation with an incorrect Euler-number.

In this paper we propose a different approach that improves on our previous work. Specifically, we continue to identify topological defects as locally noninvertible regions, but no longer use the spherical mapping to produce candidate solutions. Instead, we generate potential solutions using the concept of nonseparating loops and opening operators [39], [52]. Using this concept, the space of potential solutions is no longer restricted by the spherical mapping, resulting in a significantly more diversified set of candidate corrections, spanning a broader space. In addition, we avoid using floating-point arithmetic by working directly on the graph of the triangulation, thereby using exact arithmetic computations. Our method proceeds as follows.

1) Generate a mapping from the original cortical surface onto the sphere that is as close as possible to a homeomorphism (which was termed a maximally homeomorphic mapping or a quasi-homeomorphic mapping in [42] and in [44]). Each topological defect is then identified as a set of overlapping triangles on the sphere.

2) For each topological defect, randomly generate sets of nonseparating loops, and correct for the topology of the defect by opening and sealing the surface. The final maximum $a$ posteriori configuration is selected in a Bayesian framework.

The method is a wholly self-contained topology correction algorithm, which determines geometrically accurate and topologically correct solutions based the MRI intensity profiles and the expected local curvature.

\section{IDENTIFICATION OF TOPOLOGICAL DEFECTS}

We identify the presence of topological defects in the surface $\mathcal{C}$ by computing its Euler-characteristic ${ }^{4} \chi(\mathcal{C})$. In the presence of topological defects (i.e., $\chi \neq 2$ ), ${ }^{5}$ a mapping from the cortical surface $\mathcal{C}$ onto the sphere $\mathcal{S}$ that is homeomorphic over the majority of the manifold is generated, and we identify each defect as a set of overlapping faces. This step is identical to the approach developed by Fischl et al. in [42] and used in [44].

Briefly, identifying topological defects begins with the inflation and projection of the cortical surface $\mathcal{C}$ onto a sphere $\mathcal{S}$. The next step is to create a mapping $\mathcal{M}: \mathcal{C} \rightarrow \mathcal{S}$ by minimizing an energy functional $E_{\mathcal{M}}$ that directly penalizes regions in which the determinant of the Jacobian matrix of $\mathcal{M}$ becomes zero or negative; these regions are nonhomeomorphic regions (i.e., locally noninvertible). The final step is to identify the topological defects by regions, where the homeomorphism is broken (i.e., regions with negative determinant or, equivalently, regions with

\footnotetext{
${ }^{4}$ The Euler number of a surface is a topological invariant (For a tessellation, it can be easily computed as: $\chi=$ \#vertices - \#edges + \#faces).

${ }^{5} \chi=2$ does not ensure the accuracy of the initial surface $\mathcal{C}$ but this problem is out of the scope of this paper.
} 
overlapping faces). To clarify, the following steps in detail are taken from [42].

\section{A. Initialization of the Mapping: Spherical Inflation}

The initial mapping of the cortical surface to that of a sphere can be made by simply projecting each point of the cortical surface to the closest point on the sphere. In so doing, large regions of the initial mapping become nonhomeomorphic.

In contrast, we use a simple procedure to unfold and smooth the folded cortical surface such that it approaches the surface of a sphere whose origin is the centroid of the initial surface. The algorithm consists of iteratively updating the position of each vertex based on a smoothness force $\mathbf{F}_{S}$, and a radial spherical force $\mathbf{F}_{R}$

$$
\mathbf{x}_{k}(t+1)=\mathbf{x}_{k}(t)+\mathbf{F}_{S}(t)+\lambda_{R} \mathbf{F}_{R}(t)
$$

where $\mathbf{x}_{k}$ is the position of the $k$ th vertex at iteration number $t$ and the smoothness force $\mathbf{F}_{S}$ is given by

$$
\mathbf{F}_{S}=\frac{1}{N_{k}} \sum_{j \in N_{k}}\left(\mathbf{x}_{j}-\mathbf{x}_{k}\right)-\frac{1}{V} \sum_{i=1}^{V} \sum_{j \in N_{i}}\left(\mathbf{n}_{i} \mathbf{n}_{i}^{\prime}\right) \cdot\left(\mathbf{x}_{j}-\mathbf{x}_{i}\right)
$$

where

$$
\begin{array}{ll}
N_{k} & \text { set of vertices neighboring the } k \text { th vertex; } \\
V & \begin{array}{l}
\text { number of vertices in the tessellation; } \\
\mathbf{n}_{k} \text { and } \mathbf{n}_{k}^{\prime}
\end{array} \\
\begin{array}{l}
\text { surface normals at location } k \text { and its transpose, } \\
\text { respectively. }
\end{array}
\end{array}
$$

The smoothness term $\mathbf{F}_{S}$ moves each vertex in the direction of the centroid of its neighbors, while projecting out the average inward movement created over the entire surface. The radial term simply drives each vertex toward the surface of a sphere with the desired radius $R$

$$
\mathbf{F}_{R}=\left(\mathbf{R}_{\mathbf{k}}-\mathbf{x}_{\mathbf{k}}\right)
$$

where $\mathbf{R}_{k}$ is the radial projection of $\mathbf{x}_{k}$ onto the sphere with radius $R$.

We use an $R$ on the order of $100 \mathrm{~mm}$ to generate a sphere with roughly the same total surface area as an average cortex, and a $\lambda_{R}$ of 0.25 to allow for sufficient smoothing to take place during the spherical inflation. Once the inflation has converged, the surface is projected to lie precisely on the surface of a sphere of radius $R$, and whose origin is the centroid of the initial surface.

\section{B. Quasi-Homeomorphic Mapping}

Once the initial spherical configuration has been established, we generate a mapping $\mathcal{M}$ that is as close as possible to a homeomorphism. A mapping is a homeomorphism if the determinant of its Jacobian matrix is nonsingular, and the mapping itself is continuous. This is of course the multidimensional analog of monotonicity. In creating the mapping $\mathcal{M}$, only its topological

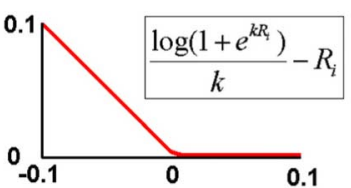

a)

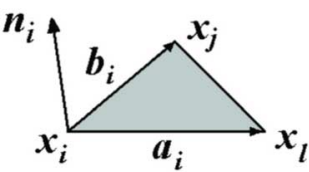

b)
Fig. 4. (a) Nonlinearity of the energy functional $E_{\mathcal{M}}$ (b) Triangle properties.

properties are concerned. To construct the mapping, we minimize an energy functional that directly penalizes regions in which the determinant becomes zero or negative, thus encouraging positive definiteness. Note that this is the only term in the energy functional-no preservation of metric properties is needed.

1) The Energy Functional: More specifically, noting that the Jacobian yields a measure of the deformation of an oriented area element under the mapping $\mathcal{M}$, the energy functional $E_{\mathcal{M}}$ constrains the penalization of compression primarily to negative semi-definite regions. If the initial area on the folded surface of the $i$ th face is $A_{i}^{0}$, and the area on the spherical surface $\mathcal{S}$ at time $t$ of the numerical integration is $A_{i}^{t}$, then the energy functional is given by

$$
\begin{aligned}
E_{\mathcal{M}} & =\sum_{i=0}^{F} \frac{1}{k} \log \left(1+e^{k R_{i}}\right)-R_{i} \text { with } R_{i}=\frac{A_{i}^{t}}{A_{i}^{0}} \\
\frac{\partial E_{\mathcal{M}}}{\partial A_{i}^{t}} & =\frac{-1}{A_{i}^{0}\left(1+e^{k R_{i}}\right)} .
\end{aligned}
$$

The logarithmic nonlinearity restricts the penalization of compression primarily to negative semi-definite regions, as can be seen in the plot in Fig. 4(a). $R_{i}$ is an approximation of the Jacobian of the transformation $\mathcal{M}, R_{i} \approx J_{\mathcal{M}}=|(\partial \mathcal{M} / \partial \mathbf{x})|$. The extent to which highly compressed positive definite regions are penalized is determined by $k$. In practice, we used a value for $k$ of 100 .

2) Numerical Implementation: In order to complete the definition of the topology term of the energy functional, we consider the $i$ th triangle in the surface tessellation, with edges $\mathbf{a}_{i}$ and $\mathbf{b}_{i}$ connecting the vertex $\mathbf{x}_{i}$ to two of its neighbors $\mathbf{x}_{l}$ and $\mathbf{x}_{j}$ respectively. In the spherical representation, the normal vector field can be given a consistent orientation on the surface ${ }^{6}$ using the embedding space, and $A_{i}$ becomes an oriented area, which may take on negative values-indicating folds in the surface. The normal vector is chosen to be pointing outward on the surface of the sphere $\mathbf{n}_{i}=\left(\mathbf{x}_{i} /\left\|\mathbf{x}_{i}\right\|\right)$ (the sphere is centered at the origin).

Using the chain rule, the directional derivative of $E_{\mathcal{M}}$ with respect to the position of the $k$ th vertex

$$
\frac{\partial E_{\mathcal{M}}}{\partial \mathbf{x}_{k}}=\frac{\partial E_{\mathcal{M}}}{\partial A_{i}^{t}} \frac{\partial A_{i}^{t}}{\partial \mathbf{x}_{k}}
$$

The first factor is given by (5). The second is the change in the area of the $i$ th triangle caused by moving the $k$ th vertex,

\footnotetext{
${ }^{6}$ This is always possible except in pathological cases, such as the Mobius strip, that are nonorientable.
} 
which can be computed from the prior description of the metric properties of the tessellation using the chain rule

$$
\frac{\partial A_{i}^{t}}{\partial \mathbf{x}_{k}}=\frac{\partial A_{i}^{t}}{\partial \mathbf{a}_{i}} \frac{\partial \mathbf{a}_{i}}{\partial \mathbf{x}_{k}}+\frac{\partial A_{i}^{t}}{\partial \mathbf{b}_{i}} \frac{\partial \mathbf{b}_{i}}{\partial \mathbf{x}_{k}}
$$

with

$$
\frac{\partial A_{i}^{t}}{\partial \mathbf{a}_{i}}=\mathbf{b}_{i} \wedge \mathbf{n}_{i}, \frac{\partial A_{i}^{t}}{\partial \mathbf{b}_{i}}=\mathbf{n}_{i} \wedge \mathbf{a}_{i} .
$$

The partials of the change in the legs with respect to a change in the vertex position are dependent on what position the vertex in question occupies in a given triangle

$$
\begin{aligned}
\frac{\partial \mathbf{a}_{i}}{\partial \mathbf{x}_{k}}= \begin{cases}{[-1,-1,-1]^{T},} & k=i \\
{[1,1,1]^{T},} & k=l \\
{[0,0,0]^{T},} & \text { otherwise }\end{cases} \\
\frac{\partial \mathbf{b}_{i}}{\partial \mathbf{x}_{k}}=\left\{\begin{array}{ll}
{[-1,-1,-1]^{T},} & k=i \\
{[1,1,1]^{T},} & k=j \\
{[0,0,0]^{T},} & \text { otherwise }
\end{array} .\right.
\end{aligned}
$$

\section{Identification of Topological Defects}

The resulting mapping $\mathcal{M}$-from the initial tessellation $\mathcal{C}$ to the sphere $\mathcal{S}$-is homeomorphic over the majority of the manifold. The surface is then examined for regions of noninvertibility, since these are the areas in which the current tessellation must be corrected to ensure proper topology. Multivalued regions, containing overlapping triangles, ${ }^{7}$ constitute topological defects where the mapping is nonhomeomorphic (these regions contain noncontractible curves, i.e., handles). $\mathcal{M}$ associates at each vertex $v$ of the initial cortical surface $\mathcal{C}$ a vertex $v_{\mathcal{S}}=\mathcal{M}(v)$ on the sphere $\mathcal{S}$. Vertices with spherical coordinates that intersect a set of overlapping triangles are marked as defective, with topological defects identified as connected sets of defective vertices.

Once a defect $\mathcal{D}$ has been identified, we compute its number of topological defects (i.e., handles). This number $g(\mathcal{D})$ is called the genus and is related to the Euler number of the defect $\chi(\mathcal{D})$ by the formula: $g(\mathcal{D})=((1-\chi(\mathcal{D})) / 2)$. An Euler number $\chi(\mathcal{D})=1$ implies that the topology of the $\operatorname{defect}^{8}$ is the one of a disk (i.e., a patch without any handles), and the defect can be discarded from the list. Note that defects having an Euler characteristic $\chi(\mathcal{D})=1$ indicates that the spherical mapping (Section III-B) is not precisely maximally homeomorphic and that the minimization of the energy functional $E_{\mathcal{M}}$ has reached a local minimum. However, while the method might mistakenly identify some topologically planar patches of surfaces that are subsequently discarded from the list, all topological defects are discovered. In our experience, the false positive rate of the spherical mapping is quite low (less than $10 \%$ of the defects are mistakenly identified).

\footnotetext{
${ }^{7}$ In practice, we detect topological defects as sets of self-intersecting edges.

${ }^{8}$ For a topological defect $\mathcal{D}$ with $n_{f}$ faces, $n_{e}$ edges, and $n_{v}$ vertices (out of which $n_{b}$ are border vertices), we always have the relations: $n_{v}-n_{e}+n_{f}=$ $\chi(\mathcal{D})$ and $n_{e}=\left(3 n_{f} / 2\right)+\left(n_{b} / 2\right)$.
}

\section{GeOMETRICAlly ACCURATE TOPOLOGY CORRECTION}

Given a topological defect, our goal is to "optimally" correct its topology, that is, to generate a defect as geometrically accurate and topologically correct as possible, given the available information. Correcting the topology amounts to finding the handles present in the defect and removing them. In algebraic topology, a handle [or, equivalently, a hole-Fig. 2(a)] in a 2-D smooth manifold is indicated by the presence of a simple closed curve that cannot be continuously deformed on the manifold into a single point. These noncontractible curves are called nonseparating loops, since they do not partition the space (i.e., the manifold) into two connected components. This concept is closely related to Morse functions, Reeb graph theory, and homotopy [45]-[47]. The removal of nonseparating loops reduces the genus of a manifold by cutting/opening the surface along the closed curves; they have been extensively used in graphics [39], [53], [54], combinatorial and computational topology [52], [55]-[58], and medical imaging [40].

Our approach is based on the concept of nonseparating loops: we correct the topology of each defect by opening and sealing the surface along an array of nonseparating loops, assessing the resulting surface for optimality with respect to a Bayesian energy functional. This method is similar to (and was inspired by) the approach proposed by Guskov and Wood in [39]. Once a nonseparating loop has been identified, we simply discard its faces and close the open mesh by attaching two pretessellated disks. We randomly generate a set of nonseparating loops and select the maximum a posteriori correction in a Bayesian framework, monitoring the accuracy as well as the validity ${ }^{9}$ of the solution. For each of the candidate solutions, an active contour optimization enhances the accuracy of the topologically corrected surface.

Algorithm 1 Geometrically Accurate Topology Correction

for all Defect $\mathcal{D}$ such that $\chi(\mathcal{D}) \neq 1$ do

for $1 \leq n \leq g(\mathcal{D})$ do

for $1 \leq i \leq$ numbers of attempts do

Random generation of a nonseparating loop $\mathcal{L}_{i}$

Mesh opening by discarding the loop faces $\mathcal{D}_{i}=\mathcal{D} \backslash \mathcal{L}_{i}$

Sealing of the mesh $\mathcal{D}_{i}$.

Smoothing and self-intersection test.

Active Contour Optimization $\mathcal{D}_{i}$.

end for

$$
\mathcal{D}=\underset{\text { valid } \mathcal{D}_{i}}{\operatorname{argmax}} p\left(\mathcal{D}_{i} \mid \mathcal{C}, I\right)
$$

\section{end for}

\section{end for}

${ }^{9}$ We ensure that the final solution has the correct topology and does not selfintersect. 

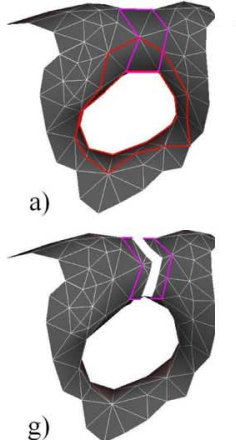
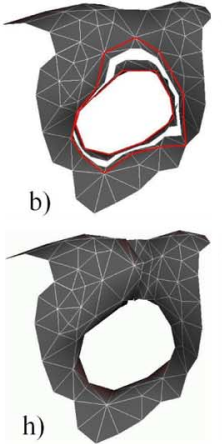
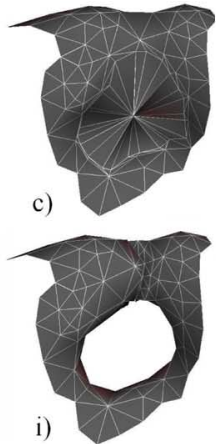
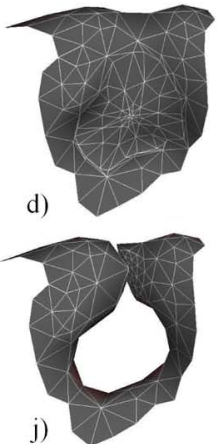
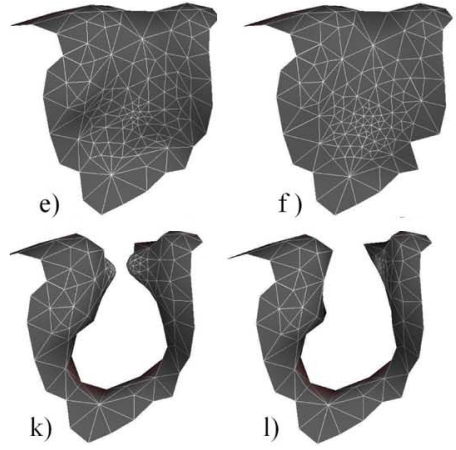

Fig. 5. (a) Identification of two associated nonseparating loops on a topological defect containing only one single handle. (b)-(f) Topological correction using the "red" nonseparating loop. Opening (b), sealing (c), and smoothing (relaxation procedure) of the two attached pretessellated disks (c)-(f). This correction amounts to filling the hole of the defect. (g)-(l) Topological correction using the "purple" nonseparating loop. This correction corresponds to cutting the handle of the defect. The "purple" loop, which is the shortest one in the defect, is the first selected one.

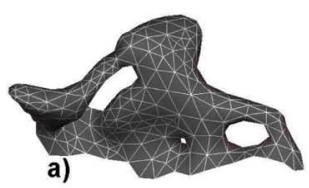

a)

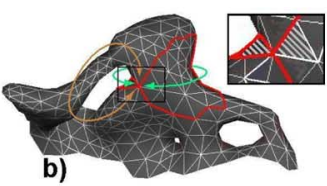

b)

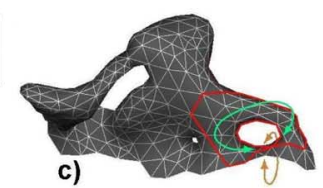

c)

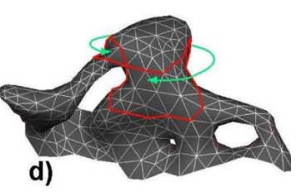

d)

Fig. 6. Nonseparating loops on an open surface. (a) Patch with three topological defects (i.e., handles). (b) The wavefront intersection is used to identify a handle on the surface. Note that the remaining faces in the defect are connected and that two associated nonseparating loops are identified: choosing the "green" loop corrects the topology by cutting the handle, while choosing the "yellow" one fills the corresponding hole. The magnified region illustrates the wavefront intersection with two triangles (dashed region) intersecting in a single vertex. (c) The front intersection indicates the potential presence of a handle in the defect. In this example, the rest of the faces are connected but not within the defect: this wavefront evolution can be used to locate only one single nonseparating loop, which, in this case, corresponds to filling the hole. We note that other wavefront evolutions (i.e., starting from other seed faces) will often produce two nonseparating loops, and not always one single nonseparating loop. (d) A wavefront intersection does not always imply that a nonseparating loop exists, as this example illustrates: the remaining faces are not connected. Therefore, we continue evolving the front.

Our method is able to produce topological corrections corresponding to cutting the handle or filling the associated hole, depending on the identified nonseparating loop. Moreover, note that filling the associated hole is equivalent to cutting the background handle identified by a nonseparating loop. Fig. 5 illustrates this point by showing two nonseparating loops that result in different topological corrections. In addition, the position of the loop (the exact path onto the triangulation) determines the shape of the final corrected surface.

\section{A. Generation of Non-Separating Loops}

In the discrete formulation a nonseparating loop is a set of connected faces that does not divide the rest of the triangulation into two connected components. ${ }^{10}$ The concept of nonseparating loops on a tessellation was introduced in the graphics community for topological noise removal by Guskov and Wood [39]. In their work, a selected vertex is used to initialize a region growing algorithm, which detects loops (i.e., topological defects) in the triangulation where wavefronts meet. Topological corrections are then obtained through the use of opening and sealing operators on the triangle mesh. While their method is fast, it depends on the initially selected vertex and does not guarantee a valid geometrically accurate surface, as such corrections may create self-intersections. In addition, their approach, as well as [40], [52], is limited to triangulations produced by a fast-marching algorithm [59], [60], while requiring a closed surface.

\footnotetext{
${ }^{10}$ For a nonseparating loop $\mathcal{L}$ on a manifold $\mathcal{C}, \chi(\mathcal{L})=0$ and $\chi(\mathcal{C})=$ $\chi(\mathcal{C} \backslash \mathcal{L})$.
}

In contrast, the method we propose is not constrained to specific types of triangulations, nor does it necessitate a closed surface. The only requirement is that the initial surface be a valid triangulation, i.e., one for which each vertex possesses only one ring of connected neighbors. Similar to the approach of Guskov and Wood, we use a local wave front to locate handles in a defect $\mathcal{D}$. We evolve a front of triangles on the triangulation until we detect a front intersection. Caution is warranted in dealing with an open surface (see Fig. 6). Given a randomly selected seed face $f_{s}$, we evolve a front of faces by fast-marching on the triangulation using approximated geodesic distances. ${ }^{11}$ At each step, we check if the candidate face induces a front intersection, detected as the intersection of two triangles, $f_{a}$ and $f_{b}$, in a single vertex [Fig. 6(b)-(d)]. Once a front intersection has been detected, we examine if the remaining faces in the triangulation are connected. In this case, we have identified a nonseparating loop in the defect. Additionally, when the remaining faces are connected inside the defect $\mathcal{D}$, a second nonseparating loop is identified [Fig. 6(b)], and we randomly select one of them. Otherwise, we resume the front evolution [Fig. 6(d)]. We note that an efficient implementation of the fast-marching method is attainable using the min-heap data structure, resulting in a complexity of $O\left(n_{f} \log n_{f}\right)$, where $n_{f}$ is the number of faces in the defect. The identification of a connected path in the remaining faces of the defect can also be achieved by fast-marching on the triangulation, starting from two neighboring faces of $f_{a}$, or $f_{b}$,

\footnotetext{
${ }^{11}$ We approximate the distance between two triangles by the Euclidean dis-
} tance in between their center of gravity. 


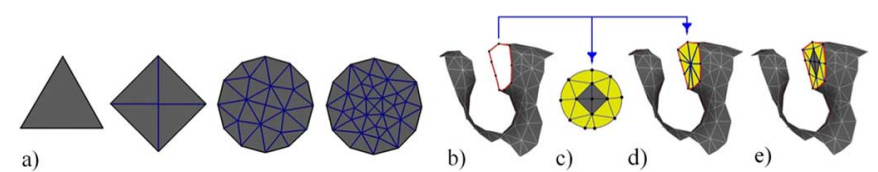

Fig. 7. (a) Examples of pretessellated disks used in the sealing process. (b) View of an open surface before attachment. (c)-(d) Attachment operation. (c) The pretessellated disk (in gray) has five vertices, regularly spaced along a unit disk $\mathcal{U}(\mathbf{0}, 1)$, and four faces. The yellow triangles are the ones that are necessary to attach the disk to the open surface. To this end, the defect vertices are spaced along $\mathcal{U}(\mathbf{0}, 2)$ proportionally to their geodesic distance on the surface and a Delaunay triangulation is generated. (d) The position of the newly attached vertices (i.e., the five vertices of the pretessellated disk) is first set to the average of the positions of the vertices constituting the loop. (e) View of the sealed surface after a relaxation procedure on the disk vertices.

since $f_{a}$ and $f_{b}$ intersects in a single vertex [magnified region of Fig. 6(b)].

Once a valid wavefront intersection has been detected, we extract a nonseparating loop (i.e., a set of connected faces) by back-tracking the faces starting from the front intersection (i.e., $f_{a}$ and $f_{b}$ ) until we reach the initial seed face $f_{s}$. We "close" the loop at the front intersection [since $f_{a}$ and $f_{b}$ intersects in a single vertex -Fig. 6(b)] by adding the shortest path of defect faces that connects $f_{a}$ to $f_{b}$. Details on the implementation can be found in [61].

\section{B. Reducing the Genus: Cutting and Sealing the Open Surface}

1) Sealing the Cut: once a nonseparating loop has been found, we discard the faces of the loop and seal the surface by attaching two pretessellated disks (i.e., patches without any topological defects) to both open sides of the defect. The attachment procedure is a graph operation designed to find a set of connecting edges and corresponding faces between two rings of vertices formed by one open side of the defect and the border of the disk. The exact shape of the surface and the pretessellated disk (i.e., the locations of its vertices) is not important in the attachment of the two disks.

To attach a pretessellated disk to one open side of the defect [Fig. 7(b)-(d)], we assume that the border vertices of the disk of the curve are regularly spaced along a unit disk $\mathcal{U}(\mathbf{0}, 1)$. We also assume that the vertices of the open side of the defect are spaced along a disk $\mathcal{U}(\mathbf{0}, 2)$ proportionally to their geodesic distance on the surface. Attaching the disk to the surface amounts to finding a triangulation from one to the other. In practice, we use a Delaunay triangulation.

For visualization purposes, we have set the position of each attached vertex to the average of the positions of the vertices constituting the loop [Figs. 5(c) and (h) and 7(b)-(d)]: the vertices of each pretessellated patch have, after attachment, the same spatial location. The size of the disk used to patch the surface is based on the size of the contour or the defect, with larger disks used to seal larger patches. Fig. 7(a) shows some typical patches.

2) Smoothing and Self-Intersection Check: The attachment operation reduces the genus of the surface by one. Although the intrinsic topology has been modified, we have not yet produced an accurate correction since all attached vertices have the same inaccurate location [the average of the positions of the loop vertices-Fig. 7(d)]. Our goal is to optimize the shape of the surface

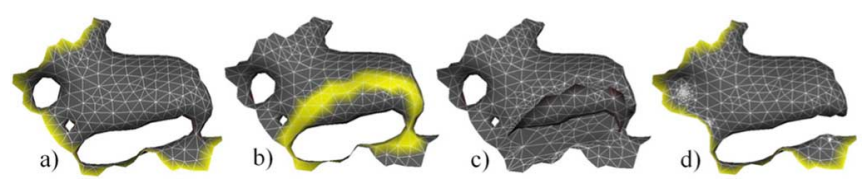

Fig. 8. (a) A complex topological defect with three handles. (b) A large nonseparating loop that leads to a self-intersecting patch (c). Such nonseparating loops are detected and discarded from the topology correction process. (d) Geometrically accurate topological correction.

by modifying the position of the defect vertices (particularly the position of the newly attached vertices) in order to maximize the goodness of fit of the corrected defect. We also need to ensure that no self-intersections are created by topological corrections. In mathematical terms, we can express this condition as

$$
\mathcal{D}_{\text {final }}=\underset{\operatorname{valid} \mathcal{D}_{i}}{\operatorname{argmax}} g\left(\mathcal{D}_{i}, I, \mathcal{C}\right)
$$

where $g\left(\mathcal{D}_{i}, I, \mathcal{C}\right)$ is a measure of the goodness of fit of the candidate solution $\mathcal{D}_{i}$, and "valid" $\mathcal{D}_{i}$ designates a nonself-intersecting patch. This continuous formulation of the problem does not translate easily into a discrete framework, in which self-intersections are difficult to detect and prevent.

For this reason, we take a somewhat different approach. After attachment, we iteratively update the positions of the newly added vertices by iteratively averaging their location with that of their neighbors (we also include in the relaxation procedure the two first neighbors of the newly attached vertices). This process is illustrated by Fig. 5. After convergence, we check if the sealed surface self-intersects and discard nonseparating loops that lead to self-intersecting surfaces [Fig. 8(b) and (c)]. We detect self-intersecting faces using a discretized (1-mm voxels) spatial look-up table to constrain the self-intersection check to faces in the local neighborhood that have been updated in the relaxation process.

The relaxation procedure is quite fast (a hundred iterations sufficient to achieve convergence), and most of the time yields a valid solution, i.e., a patch that does not self-intersect. In our experience, less than $5 \%$ of the patches self-intersect. Fig. 9 provides some examples of valid candidate patches that have had their genus reduced by one (i.e., candidate patches with one handle less than the initial patch). Note the wide range of potential solutions. Finally, once a valid topological correction is obtained, we improve the accuracy of the correction by maximizing a fitness function $g(\mathcal{D}, I, \mathcal{C})$ defined in Section IV-C, using an active contour formulation.

\section{Fitness and Likelihood Functions}

Many valid topological corrections exist (Fig. 9), and one would like to select the solution that maximizes both the goodness of fit of the final surface given the available image information, as well as one that conforms to our prior knowledge about the cortex. A cortical surface is a smooth manifold $\mathcal{C}$ that partitions the embedding space into an interior, composed of WM (as well as deep gray structures and ventricles far from the surface), and an exterior, composed of GM. We characterize the goodness of a retessellation by measuring the following two: 

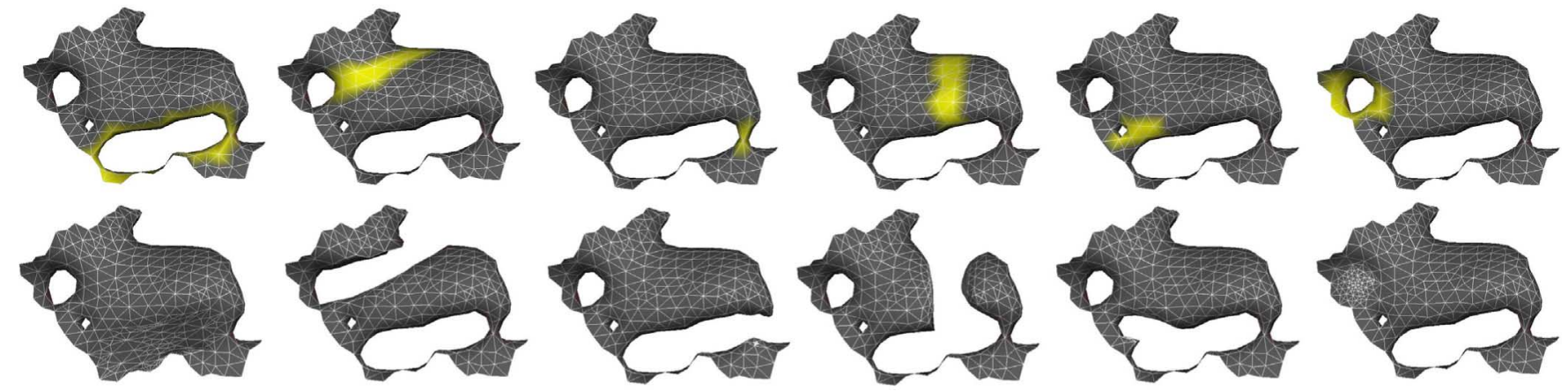

Fig. 9. Examples of nonseparating loops (top row) with their associated topological correction (bottom row). Note the wide range of potential solutions that can be generated (before active contour matching).

1) The smoothness of the resulting surface,

2) the MRI values $I$ inside and outside the surface.

We define the fitness function as the posterior probability of observing the corrected defect $\mathcal{D}_{i}$ given the MRI intensity values and the surface $\mathcal{C}: g\left(\mathcal{D}_{i}, I, S\right)=p\left(\mathcal{D}_{i} \mid \mathcal{C}, I\right)$. Formally, the posterior probability of the $i$ th retessellation $\mathcal{D}_{i}$ is given by

$$
p\left(\mathcal{D}_{i} \mid \mathcal{C}, I\right) \propto p\left(I \mid \mathcal{C}, \mathcal{D}_{i}\right) p\left(\mathcal{D}_{i} \mid \mathcal{C}\right) .
$$

The likelihood term $p\left(I \mid \mathcal{C}, \mathcal{D}_{i}\right)$ encodes information about the MRI intensities inside and outside the surface. Each corrected defect separates the underlying MRI volume into two distinct components, ${ }^{12}$ an inside part $\mathcal{C}^{-}$and an outside part $\mathcal{C}^{+}$. An acceptable candidate solution should create a space partition with the majority of its inside and outside voxels corresponding to WM and GM voxels, respectively. In order to estimate the likelihood $p\left(I \mid \mathcal{C}, \mathcal{D}_{i}\right)$, we assume that the noise is spatially independent. This probability can then be rewritten as

$$
\begin{aligned}
& p\left(I \mid \mathcal{C}, \mathcal{D}_{i}\right)= \underbrace{}_{\text {volume -based information }} p_{w}\left(I(x) \mid \mathcal{C}, \mathcal{D}_{i}\right) \prod_{x \in \mathcal{C}^{+}} p_{g}\left(I(x) \mid \mathcal{C}, \mathcal{D}_{i}\right) \\
& \underbrace{\prod_{v=1}^{V_{i}} p\left(g_{i}(v), w_{i}(v) \mid \mathcal{C}, \mathcal{D}_{i}\right)}_{\text {surface- based information }}
\end{aligned}
$$

where $p_{w}\left(I(x) \mid \mathcal{C}, \mathcal{D}_{i}\right)$ and $p_{g}\left(I(x) \mid \mathcal{C}, \mathcal{D}_{i}\right)$ are the likelihood of intensity values at location $x$ in the volume inside and outside the tessellation respectively, $p\left(g_{i}(v), w_{i}(v) \mid \mathcal{C}, \mathcal{D}_{i}\right)$ is the joint likelihood of intensity values inside and outside the tessellation at vertex $v$ in tessellation $\mathcal{D}_{i}$.

Geometric information can be incorporated via $p\left(\mathcal{D}_{i} \mid \mathcal{C}\right)$, which represents priors on the possible retessellation. For example, $p\left(\mathcal{D}_{i} \mid \mathcal{C}\right)$ could have the form

$$
p\left(\mathcal{D}_{i} \mid \mathcal{C}\right)=\prod_{v=1}^{V_{i}} p\left(\kappa_{1}(v), \kappa_{2}(v) \mid \mathcal{C}\right)
$$

${ }^{12}$ We use the angle-weighted pseudonormal algorithm [62] to compute the signed distance of the tessellation. The voxel grid is partitioned into inside negative values and outside positive values where $\kappa_{1}$ and $\kappa_{2}$ are the two principal curvatures of the surface, computed at vertex $v$. In our experience, this choice of priors produces accurate candidate solutions.

Given that the vast majority of the surface is in general nondefective, we have an ample amount of data to estimate the correct forms of the distributions $p\left(\mathcal{D}_{i} \mid \mathcal{C}\right), p_{g}\left(I(x) \mid \mathcal{C}, \mathcal{D}_{i}\right)$, $p_{w}\left(I(x) \mid \mathcal{C}, \mathcal{D}_{i}\right)$, and $p\left(g_{i}, w_{i} \mid \mathcal{C}, \mathcal{D}_{i}\right)$. In particular, the single tissue distributions $p_{g}\left(I(x) \mid \mathcal{C}, \mathcal{D}_{i}\right)$ and $p_{w}\left(I(x) \mid \mathcal{C}, \mathcal{D}_{i}\right)$ are estimated locally around each topological defect, in a region that excludes the defect itself (the region is taken as a cubic box containing the defect $\pm 5 \mathrm{~mm}$ ). This makes the procedure completely adaptive and self-contained, in the sense that no assumptions need to be made about the contrast of the underlying MRI image(s), and no training or parametric forms are required for $p\left(\mathcal{D}_{i} \mid \mathcal{C}\right)$. An example of the estimation of $p\left(g_{i}, w_{i} \mid \mathcal{C}, \mathcal{D}_{i}\right)$ and $p\left(\mathcal{D}_{i} \mid \mathcal{C}\right)$ is given in Fig. 10. Fig. 10(b) shows the joint distribution of GM and WM given the surface computed, using the nondefective portion of the gray/white boundary representation of a single subject. Note the diagonal character of the distribution that indicates the mutual dependence of the intensities-brighter WM typically means brighter GM due to factors such as bias fields induced by RF inhomogeneities and coil sensitivity profiles, as well as intrinsic tissue variability. One possible form of the priors on the tessellation is given by Fig. 10(c), which shows the joint distribution of the two principal curvatures $\kappa_{1}$ and $\kappa_{2}$ computed over the nondefective portion of a single surface.

\section{Optimization Using Active Contour Patches}

During the search, candidate patches $\mathcal{D}_{i}$ are selected based on their fitness value $p\left(\mathcal{D}_{i} \mid \mathcal{C}, I\right)$. After attachment, smoothing, and the self-intersection check, each patch defines a valid manifold that can be treated as an active contour with fixed boundaries. Each patch is locally deformed in order to maximize the posterior probability $p\left(\mathcal{D}_{i} \mid \mathcal{C}, I\right)$. Instead of deriving the exact Euler-Lagrange equation of the active contour $\mathcal{D}_{i}$ for the energy functional $p\left(\mathcal{D}_{i} \mid \mathcal{C}, I\right)$, we use an approximation procedure. We note that the fitness function of a candidate solution measures the smoothness of the resulting surface and the MRI intensity profile inside and outside the surface. We simply update the position of each interior vertex $\mathbf{x}_{k}$ of the candidate tessellation 

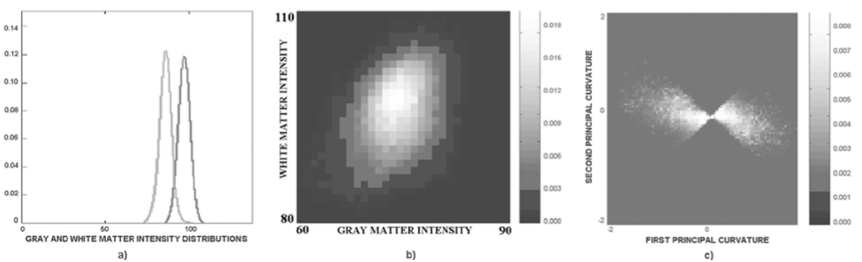

Fig. 10. (a) Examples of the GM and WM distributions estimated locally from a given a topological defect. (b) Joint distribution of GM and WM given the surface computed, using the nondefective portion of the gray/white boundary representation of a single subject. The GM and WM intensity are two correlated variables, as indicated by the diagonal structure of the joint distribution. (c) Joint distribution of two principal curvatures of the surface.

based on a smoothness force $\mathbf{F}_{S}$ and an MRI intensity-based force $\mathbf{F}_{M}$

$$
\mathbf{x}_{k}(t+1)=\mathbf{x}_{k}(t)+\mathbf{F}_{S}(t)+\lambda_{M} \mathbf{F}_{M}(t) .
$$

The smoothness force is the same as the one defined in (2). The intrinsic curvature-based force enforces a smoothness constraint on the deformed active contours and tends to maximize the prior term $p\left(\mathcal{D}_{i} \mid \mathcal{C}\right)$. The MRI intensity-based force $\mathbf{F}_{M}$ is designed to drive the active contour towards the true boundary separating the GM from the WM

$$
\mathbf{F}_{M}=\left[T_{v}-I\left(\mathbf{x}_{k}\right)\right] \nabla I\left(\mathbf{x}_{k}\right)
$$

where the targeted value $T_{v}$ is computed from the GM and WM distributions. The mean intensity and variance of the GM and WM intensities are estimated from the respective distributions $p_{g}$ and $p_{w}$, denoted by $\mu^{g}, \sigma^{g}, \mu^{w}$, and $\sigma^{w}$, and the local threshold $T_{v}$ is computed using the following equation:

$$
T_{v}=\frac{\mu^{w} \sigma^{g}+\mu^{g} \sigma^{w}}{\sigma^{w}+\sigma^{g}} .
$$

At each iteration, we measure the exact fitness function $p\left(\mathcal{D}_{i} \mid \mathcal{C}, I\right)$ of the active contour and stop the deformation when the fitness function is maximized. We ensure that the surface remains a valid one by preventing self-intersections during the active contour optimization. The constant $\lambda_{M}$ is empirically set to 0.5 .

\section{E. Implementation Parameters}

The proposed approach is implemented using the following parameters. A typical topological defect contains on the order of 100 faces. For a defect containing $n_{f}$ faces, we produce $n_{f} / 3$ patches per handle by generating nonseparating loops from "semi-randomly" selected seed faces. We use spatial information to better distribute randomly selected faces inside the defect by ensuring that a seed face is not drawn twice and that a new draw is not neighboring the previous one. This procedure produces random selection of seed faces that are likely to cover the defect uniformly. As such, our method samples likely solutions with high probability. In addition, the first nonseparating loop is always the smallest loop in the defect. To identify the smallest loop, we simply generate as many loops as there are faces in the defect and select the shortest one. Note that the loop generation is computationally fast (in $O\left(n_{f} \log n_{f}\right)$ ), while detecting self-intersections and the computation of the fitness function are the slowing factors.

\section{VALIDATION}

We have applied our proposed approach to 43 real images (this data set is the same one used for the evaluation of our previous method [44]). The dataset is comprised of MRI volumes of differing quality, from varying populations (both pathological and healthy) scanners (1.5T GE and Siemens), and pulse sequences (MP-RAGE and SPGR). Results were evaluated by experts to assess the accuracy of the final corrections.

\section{A. Description of the Data Set}

Validation data came from several data sets. They were a mix of pulse sequence (SPGR, MP-RAGE), scanner types (Siemens 1.5T, GE 1.5T) and pathology (normal control, schizophrenia and Alhzeimer's).

Seventeen scans were acquired in 2000/2001 using a Siemens Sonata system with the following parameters: TR: $7.25 \mathrm{~ms}$; TE: $3.22 \mathrm{~ms}$; TI: $600.00 \mathrm{~ms}$; flip angle: $7.00^{\circ}$; $1.3-\mathrm{mm}$ sections (resampled to $1 \mathrm{~mm}$ isotropic). This data set consists of eight young (YNC), seven elderly normal controls (ENC), and two Alzheimer's (AD).

The second data set was acquired using a Siemens Vision system in 1994/1995 with the following parameters: TR: 9.70 ms; TE: $4.00 \mathrm{~ms}$; TI: $621.00 \mathrm{~ms}$; flip angle: $10.00^{\circ} ; 1.25-\mathrm{mm}$ sections (resampled to $1-\mathrm{mm}$ isotropic). Data comes from studies reported in Buckner et al. [63] and Logan et al. [64] and also later subjects imaged using the same anatomic protocol. ${ }^{13}$ This data set consists of six Young Normal Control, 14 nondemented and six demented adults.

\section{B. Discussion of the Results}

1) Accuracy: methods that do not integrate statistical and geometric information will often fail to produce solutions comparable to those that a trained operator makes. This is illustrated in Fig. 11(a) and (b), where valid solutions do not always correspond to minimal corrections [i.e., cutting the handles in the magnified examples of Fig. 11(a) and (b)]. Only general approaches that integrate additional information can produce correct solutions.

In order to assess the accuracy of the proposed method, we rely on experts to evaluate the solutions. All solutions matched the ones produced by a trained expert. Then, we compare our results with the ones obtained in [44]. To our knowledge, these two methods are the only ones proposed to explore the space of potential solutions for selecting the best correction to a topological defect. In most cases, both approaches produced almost-identical, correct solutions that other methods failed to produce. However, for some complex topological defects, our approach outperformed the genetic-search approach. As we previously

\footnotetext{
${ }^{13}$ We thank Randy Buckner and the Washington University Alzheimer's Disease Research Center for providing the data set.
} 

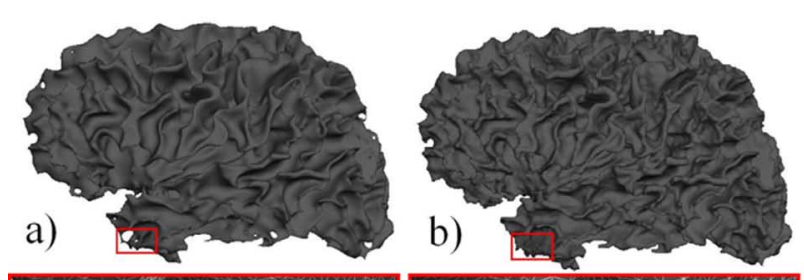

c)
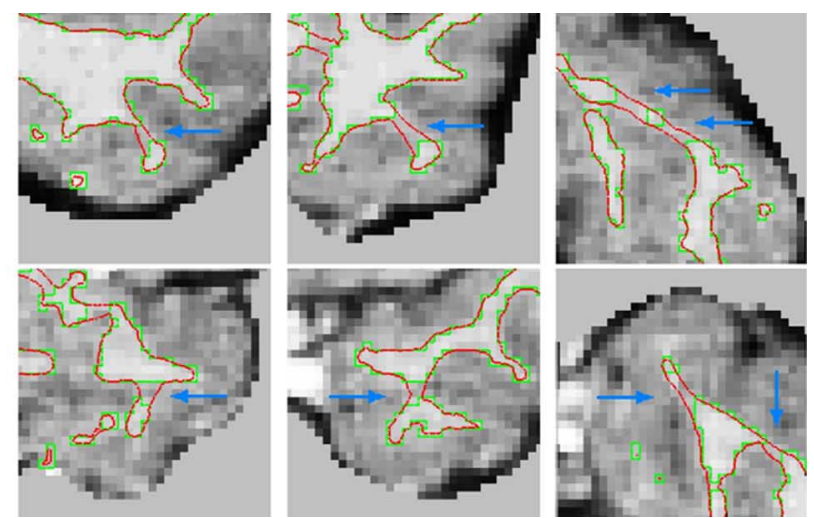

Fig. 11. (a) Original cortical surface with a magnified region. (b) Topologically corrected surface and corresponding magnified regions. (c) Coronal, horizontal, and sagittal MRI view of the cortical surfaces before (in green) and after (in red) topology correction. The topological inconsistencies correspond to the magnified region in (a) and (b). (d) Another example of a topological correction.

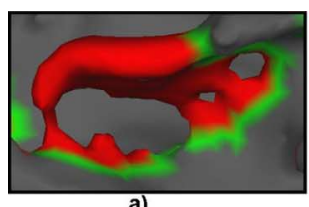

a)

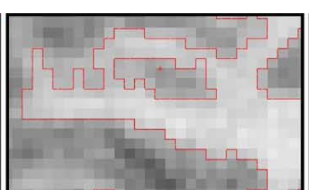

b)

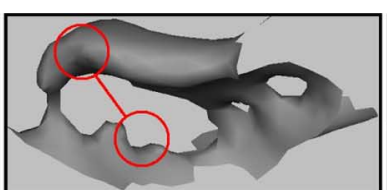

c)

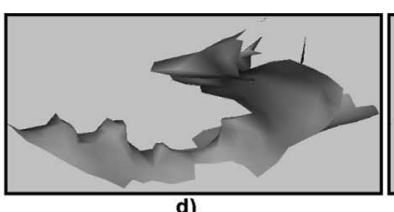

d)

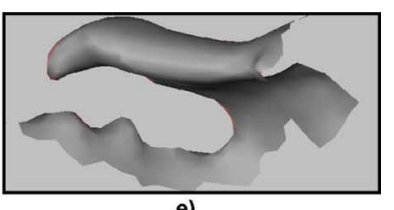

e)

Fig. 12. (a) Original defect: red and green vertices represent inside and border vertices respectively. (b) One sagittal view of the defect. (c) Original defect. The vertices in the circled regions have by the spherical mapping the same location on the sphere. (d) Incorrect solution generated by the genetic algorithm using the spherical mapping. This solution corresponds to the best candidate within the space of potential retessellation constrained by the initial spherical mapping. (e) Solution generated by our approach. One can check the similarity of our solution with the one generated by a trained operator in Fig. 3(c).

noted, the space of potential retessellation explored by the genetic algorithm depends strongly on the initial spherical mapping. For some large and complex topological defects, the genetic search does not develop correct solutions (i.e., the ones that a trained operator produces). The current approach, which does not rely on the spherical mapping to obtain candidate solutions, is more likely to generate the correct ones, as Fig. 12 illustrates.

Finally, to evaluate the quality of the corrections, we compute for each defect the average Hausdorff distance between automatically corrected surfaces (using our method) and manually corrected surfaces produced by a trained operator. The average Hausdorff distance is less than $0.2 \mathrm{~mm}$ (similar to the results obtained in our previous method [44]).

2) Numerical Implementation and Computational Time: An average cortical surface contains on the order of 50 topological defects, most of which are relatively small: most defects contain less than 50 vertices (approximately 100 faces), ${ }^{14}$ and are corrected in a few seconds.

Larger defects, with more than 100 vertices, can take a few minutes, due to the self-intersection check and the computation of the fitness associated with each defect. However, the overall process is no longer quadratic in the number of vertices contained in the convex hull of each defect, as in our previous method [44], and a full brain is corrected in approximately 20 min on a 1-GHz Pentium IV machine. ${ }^{15}$ More importantly, we stress that the whole process could be parallelized, as each de-

\footnotetext{
${ }^{14}$ For a topological defect with $g$ handles and $n_{v}$ vertices out of which $n_{b}$ are border vertices, the number of faces is $n_{f}=2\left(n_{v}-1+2 g\right)-n_{b}$

${ }^{15}$ This time estimate disregards the generation of the spherical mapping which takes itself approximately $20 \mathrm{~min}$.
}

fect is independent of one another, thus providing the potential for two order of magnitude increase in the speed of the procedure with parallelization.

In addition, our approach produces few self-intersecting patches (less than $5 \%$ of all generated patches). Most of the computation time is taken up by the calculation of the fitness and the self-intersection test, with a complexity approximately proportional to the size of the defect.

Finally, we emphasize that all of the topological operations (i.e., operations that lead to the correction of the topology) are exact arithmetic operations that manipulate the graph of the triangulation. In contrast to our previous genetic search approach that used the spherical mapping to constrain the topology of the final solution, and as such was sensitive to floating-point rounding errors, the current method always produces a valid final surface, i.e., one with the Euler-characteristic of a sphere $\chi=2$.

3) Limitations: It is important to state the limitations of the proposed approach. While the current algorithm is no longer constrained by the spherical mapping, not all configurations can be attained. The obvious explanation is the local behavior of our topological corrections, which, having identified potential cuts, optimize the shape of the surface locally by using a gradient descent within the active contour framework. Therefore, our approach may not be able to produce geometrically accurate solutions in the event of extremely noisy MRI images.

However, in our experience such configurations are quite unlikely. Topological inconsistencies are often the result of very few mislabeled ambiguous voxels during the segmentation process, and local topological corrections are usually sufficient to produce geometrically accurate solutions. While aware of 
these limitations, we do not believe this to be a real restriction to the geometrically accurate topology correction of cortical surfaces from standard structural MRI acquisitions.

\section{CONCLUSION}

We have proposed an automated method to accurately correct the topology of cortical representations. Our approach integrates statistical and geometric information in selecting the best correction for each defect. Non-separating loops locate handles present in the volume, and produce topologically corrected candidate solutions by discarding the faces that form the loops and by sealing the open mesh. The accuracy of each candidate solution is then maximized by active contour optimization. Finally, randomly generated candidate solutions are selected based on their goodness of fit in a Bayesian framework. The fitness of a retessellation is measured by the smoothness of the resulting surface and the local MRI intensity profiles inside and outside the surface. The resulting procedure is a wholly adaptative and self-contained topology correction algorithm, which determines geometrically accurate, topologically correct solutions based on the MRI intensity profiles and the expected local curvature.

The topology correction is fast, and a full brain can be corrected in about $20 \mathrm{~min}$ on a current (1-GHz Pentium IV) machine (approximately $40 \mathrm{~min}$ taking into account the generation of the spherical mapping). Exact arithmetic operations on the graph of the triangulation ensure that no floating-point rounding errors occur during the topology-correction process, at the same time guaranteeing that the final surface exhibits the topology of a sphere. To our knowledge, this approach has been the only one proposed thus far to explore the full space of potential solutions in order to select the best correction of a topological defect.

Finally, note that the proposed approach is not restricted to spherical topologies, and can be used to correct the planar topology of any triangulations.

This algorithm is part of the cortical surface reconstruction and flattening software Freesurfer, associated with [3], [5], [6], [10]. Source code for the generation of nonseparating loops, the opening and the sealing of topological defects is available at http://cermics.enpc.fr/ segonne/research/topologycorrection/.

\section{ACKNOWLEDGMENT}

This paper is part of the National Alliance for Medical Image Computing (NAMIC), funded by the National Institutes of Health through the NIH Roadmap for Medical Research under Grant U54 EB005149. Information on the National Centers for Biomedical Computing can be obtained from http://nihroadmap.nih.gov/bioinformatics.

\section{REFERENCES}

[1] A. M. Dale and M. I. Sereno, "Improved localization of cortical activity by combining EEG and MEG with MRI cortical surface reconstruction: A linear approach," J. Cognit. Neurosci., vol. 5, no. 2, pp. 162-176, 1993.

[2] D. Van Essen and H. Drury, "Structural and functional analyses of human cerebral cortex using a surface-based atlas," J. Neurosci., vol. 17, no. 18, pp. 7079-7102, 1997.
[3] A. Dale, B. Fischl, and M. Sereno, "Cortical surface-based analysis I: Segmentation and surface reconstruction," NeuroImage, vol. 9, pp. 179-194, 1999.

[4] H. Drury, D. Van Essen, C. Anderson, C. Lee, T. Coogan, and J. Lewis, "Computerized mappings of the cerebral cortex: A multiresolution flattening method and a surface-based coordinate system," J. Cognit. Neurosci., vol. 8, no. 1, pp. 1-28, 1996.

[5] B. Fischl, M. Sereno, R. Tootell, and A. Dale, "High-resolution intersubject averaging and a coordinate system for the cortical surface," Hum. Brain Mapp., vol. 8, pp. 272-284, 1999.

[6] B. Fischl and A. Dale, "Measuring the thickness of the human cerebral cortex from magnetic resonace images," Proc. Nat. Acad. Sci., vol. 97, pp. 11044-11049, 2000.

[7] J. Tanabe, D. Amend, N. Schuff, V. DiSclafani, F. Ezekiel, D. Norman, G. Fein, and M. Wiener, "Tissue segmentation of the brain in alzeihmer's disease," J. Neuroradiol., vol. 18, pp. 115-123, 1997.

[8] R. Desikan et al., "A computer generated labeling system for subdividing the human cerebral cortex on MRI scans into gyral based regions of interest," Hum. Brain Mapp., 2005.

[9] D. Salat, R. Buckner, A. Snyder, D. Greve, R. Desikan, E. Busa, J. Morris, A. Dale, and B. Fischl, "Thinning of the cerebral cortex in aging," Cereb. Cortex, vol. 14, no. 7, pp. 721-730, Jul. 2004.

[10] B. Fischl, D. Salat, E. Busa, M. Albert, M. Dieterich, C. Haselgrove, A. V. d. Kouwe, R. Killinay, D. Kennedy, S. Klaveness, A. Montillo, N. Makris, B. Rosen, and A. Dale, "Whole brain segmentation: Automated labeling of neuroanatomical strucutres in the human brain," Neuron, vol. 33, pp. 341-355, 2002.

[11] R. Kikinis et al., "Temporal love sulco-gyral pattern anomalies in schizophrenia: An in vivo MR three-dimensional surface rendering study," Neurosci. Lett., vol. 182, pp. 7-12, 1994.

[12] P. Thompson, J. Moussai, S. Zohoori, A. Goldkorn, A. Khan, M. Mega, G. Small, J. Cummings, and A. Toga, "Cortical variability and asymmetry in normal aging and alzheimer's disease," Cereb. Cortex, vol. 8, no. 6, pp. 492-509, 1998.

[13] P. Thompson, D. MacDonald, M. Mega, C. Holmes, A. Evans, and A. Toga, "Detection and mapping of abnormal brain structure with a probabilistic atlas of cortical surfaces," J. Comput. Assist. Tomogr., vol. 21, no. 4, pp. 567-581, 1998.

[14] X. Tao, X. Han, M. Rettmann, J. Prince, and C. Davatzikos, "Statistical study on cortical sulci of human brains," Proc. Information Processing in Medical Imaging, pp. 37-49, 2001.

[15] M. Vaillant and C. Davatzikos, "Hierarchical matching of cortical features for deformable brain image registration," Proc. Information Processing in Medical Imaging, pp. 182-195, 1999.

[16] C. Xu, D. L. Pham, and J. L. Prince, "Medical image segmentation using deformable models," in Handbook of Medical Imaging. Bellingham, WA: SPIE, 2000, vol. 2, Medical Image Processing and Analysis, pp. 129-174.

[17] J. Bezdek, L. Hall, L. Clarke, and Clarke, "Review of MR image segmentation techniques using pattern recognition," Med. Phys., vol. 20, pp. 1033-1048, 1993.

[18] D. Pham, C. Xu, and J. Prince, "Current methods in medical image segmentation," Annu. Rev. Biomed. Eng., vol. 2, pp. 315-337, 2000.

[19] F. Ségonne, Segmentation of medical images under topological constraints Massachusetts Inst. Technol.. Cambridge, 2005, Ph.D. dissertation.

[20] M. Kass, A. Witkin, and D. Terzopoulos, "Snakes: Active contour models," in Proc. 1st Int. Conf. Computer Vision, London, Jun. 1987, pp. 259-268.

[21] D. MacDonald, N. Kabani, D. Avis, and A. Evens, "Automated 3D extraction of inner and outer surfaces of cerebral cortex from MRI," Neurolmage, vol. 12, pp. 340-356, 2000.

[22] T. McInerney and D. Terzopolos, "Deformable models in medical image analysis: A survey," Med. Imag. Anal., vol. 1, no. 2, pp. 91-108, 1996.

[23] T. McInerney and D. Terzopoulos, "T-snakes: Topology adaptive snakes," Med. Image Anal. vol. 4, pp. 73-91, 2000. [Online]. Available: http://citeseer.nj.nec.com/mcinerney99tsnakes.html..

[24] J.-O. Lachaud and A. Montanvert, "Deformable meshes with automated topology changes for coarse-to-fine 3D surface extraction," Med. Image Anal., vol. 3, no. 2, pp. 187-207, 1999.

[25] H. Delingette, "General object reconstruction based on simplex meshes," Int. J. Comput. Vis., vol. 32, no. 2, pp. 111-146, 1999.

[26] H. Delingette and J. Montagnat, "Shape and topology constraints on parametric active contours," Comput. Vis. Image Understanding, vol. 83, no. 2, pp. 140-171, 2001. 
[27] V. Caselles, R. Kimmel, and G. Sapiro, "Geodesic active contours," Int. J. Comput. Vis., vol. 22, no. 1, pp. 61-79, 1997.

[28] X. Han, C. Xu, and J. Prince, "A topology preserving level set method for geometric deformable models," IEEE Trans. Pattern Anal. Mach. Intell., vol. 25, no. 6, pp. 755-768, Jun. 2003.

[29] F. Ségonne, J.-P. Pons, E. Grimson, and B. Fischl, "Active contours under topology control in genus preserving level sets," in Proc. Workshop Computer Vision for Biomedical Image Applications: Current Techniques and Future Trends, 2005, pp. 135-145.

[30] J.-F. Mangin, V. Frouin, I. Bloch, J. Regis, and J. Lopez-Krahe, “From 3D magnetic resonance images to structural representations of the cortex topography using topology preserving deformations," J. Math. Imag. Vis., vol. 5, pp. 297-318, 1995.

[31] F. Poupon, J.-F. Mangin, D. Hasboun, C. Poupon, I. Magnin, and V. Frouin, Multi-object deformable templates dedicated to the segmentation of brain deep structures, ser. Lecture Notes in Computer Science. Berlin, Germany: Springer-Verlag, 1998, vol. 1496, pp. 1134-1143.

[32] S. Bischoff and L. Kobbelt, "Isosurface reconstruction with topology control," Pacific Graphics Proc., pp. 246-255, 2002.

[33] P.-L. Bazin and D. L. Pham, "Topology correction using fast marching methods and its application to brain segmentation," in Proc. MICCAI (2), 2005, pp. 484-491.

[34] G. Christense, R. Rabbitt, and M. Miller, "3D brain mapping using a deformable neuroanatomy," Phys. Med. Biol., vol. 39, pp. 609-618, 1994.

[35] B. Karaça and C. Davatzikos, "Topology preservation and regularity in estimated deformation fields," in Proc. IPMI, 2003, pp. 426-437.

[36] P. Bazin and D. Pham, "Topology preserving tissue classification with fast marching and topology templates," in Proc. IPMI, 2005, pp. 234-245.

[37] D. Shattuck and R. Leahy, "Automated graph based analysis and correction of cortical volume topology," IEEE Trans. Med. Imag., vol. 20, no. 11, pp. 1167-1177, Nov. 2001.

[38] X. Han, C. Xu, U. Braga-Neto, and J. Prince, "Topology correction in brain cortex segmentation using a multiscale, graph-based approach," IEEE Trans. Med. Imag., vol. 21, no. 2, pp. 109-121, Feb. 2001.

[39] I. Guskov and Z. Wood, "Topological noise removal," in Proc. Graphics Interface, 2001, pp. 19-26.

[40] S. Jaume, Topology Simplification Algorithm for the Segmentation of Medical Images Belgium, University of Louvain, 2004, Ph.D.

[41] N. Kriegeskorte and R. Goeble, "An efficient algorithm for topologically segmentation of the cortical sheet in anatomical MR volumes," NeuroImage, vol. 14, pp. 329-346, 2001.

[42] B. Fischl, A. Liu, and A. Dale, "Automated manifold surgery: Constructing geometrically accurate and topologically correct models of the human cerebral cortex," IEEE Trans. Med. Imag., vol. 20, no. 1, pp. 70-80, Jan. 2001.

[43] F. Ségonne, E. Grimson, and B. Fischl, "Topological correction of subcortical segmentation," Proc. Medical Image Computing and Computer-Assited Intervention, vol. 2879, no. 2, pp. 695-702, 2003.

[44] — Proceedings of Information Processing in Medical Imaging, ser. Lecture Notes in Computer Science. Berlin, Germany: Springer-Verlag, 2005, vol. 3565, pp. 393-405.

[45] J. Munkres, Topology: A First Course. Englewood Cliffs, N.J.: Prentice-Hall, 1975.
[46] J. Stillwell, Classical Topology and Combinatorial Group Theory. New York: Springer-Verlag, 1993.

[47] A. Hatcher, Algebraic Topology. Cambridge, U.K.: Cambridge Univ. Press, 2002.

[48] Clay Mathematics Institute-Millennium Problems [Online]. Available: http://www.claymath.org/millennium/

[49] G. Perelman, The Entropy Formula for the Ricci Flow and its Geometric Applications 2002 [Online]. Available: http://www.claymath. org/millennium/

[50] _ Ricci Flow With Surgery on Three-Manifolds 2003 [Online]. Available: http://www.claymath.org/millennium/

[51] _ - Finite Extinction Time for the Solutions to the Ricci Flow on Certain Three-Manifolds 2003 [Online]. Available: http://www.claymath.org/millennium/

[52] A. Verroust and M. Finiasz, "A control of smooth deformations with topological change on a polyhedral mesh," in Proc. Shape Modeling Int., 2002, 2002, pp. 191-198.

[53] Z. Wood, H. Hoppe, M. Desbrun, and P. Schroder, Isosurface Topology Simplification 2002 [Online]. Available: citeseer.ist.psu.edu/wood02isosurface.html

[54] X. Gu and S.-T. Yau, "Global conformal surface parameterization," in SGP '03: Proc. 2003 Eurographics/ACM SIGGRAPH Symp. Geometry Processing, Aire-la-Ville, Switzerland, 2003, pp. 127-137.

[55] K. Cole-Mclaughlin, H. Edelsbrunner, J. Harer, V. Natarajan, and V. Pascucci, Loops in Reeb Graphs of 2-Manifolds 2003 [Online]. Available: citeseer.ist.psu.edu/article/cole-mclaughlin03loops.html

[56] F. Lazarus, M. Pocchiola, G. Vegter, and A. Verroust, "Computing a canonical polygonal schema of an orientable triangulated surface," in SCG '01: Proc. 17th Annu. Symp. Computational Geometry, 2001, pp. 80-89.

[57] E. W. Chambers, É. C. de Verdière, J. Erickson, F. Lazarus, and K. Whittlesey, "Splitting (complicated) surfaces is hard," in Proc. 22nd Annu. ACM Symp. Computational Geometry, 2006, pp. 421-429.

[58] É. C. de Verdière and F. Lazarus, "Optimal system of loops on an orientable surface," Discrete Computational Geometry, vol. 33, no. 3, pp. 507-534, Mar. 2005.

[59] W. Lorensen and H. Cline, "Marching cubes: A high-resolution 3D surface reconstruction algorithm," ACM Comput. Graphic., vol. 21, no. 4, pp. 163-170, 1987.

[60] B. K. Natarajan, "On generating topologically consistent isosurfaces from uniform samples," The Visual Comput., vol. 11, no. 1, pp. 52-62, 1994.

[61] F. Ségonne, J. Pacheco, and B. Fischl, Geometrically-Accurate Topology Simplification of Triangulated Cortical Surfaces Using Non-Separating Loops CERTIS, 2006, Certis memo RR 06-24.

[62] J. A. Baerentzen, "Signed distance computation using the angle weighted pseudonormal," IEEE Trans. Visual. Comput. Graphics, vol. 11, no. 3, pp. 243-253, May-Jun. 2005.

[63] R. Buckner, A. Snyder, A. Sanders, M. Raichle, and J. Morris, "Functional brain imaging of young, nondemented, and demented older adults," J. Cognit. Neurosci., vol. 12, no. 2, pp. 24-34, 2000.

[64] J. Logan, A. Sanders, A. Snyder, J. Morris, and R. Buckner, "Underrecruitment and nonselective recruitment: Dissociable neural mechanisms associated with agin," Neuron, vol. 33, pp. 827-840, 2002. 